DOI: https://doi.org/10.24297/jap.v16i1.8270

\title{
Nature of The Atom
}

By Leonard Van Zanten - Riverside Ca. 92505

lenvanzanten@msn.com

\begin{abstract}
The atom as a subject is the most difficult of all subjects and for its many variations extremely large and the most incomprehensive of all phenomena. Nor so I fear will we ever come to a full understanding of it, nor even the half thereof. Yet we must make a start somewhere to learn the principle fashion of its nature and operation that for what I have seen is mostly misunderstood.
\end{abstract}

I now am not enabled to reveal what exactly the atom is, nor how it precisely operates under all conditions, but I am able to grant us a more realistic look into the nature of the atomic nature.

\section{Keywords}

Motion, Magnetic, Atom,

\section{Essay}

In order to categorize what we are to discuss, first let us get into the nature of the atom, then its nomenclature, and how it forms molecules. And we must speak of the cornerstone, termed energy, as well as motion, and how by the mere rate of movements atoms and molecules shift their polarities, and how by some for a higher rate of movement molecules disband while others unite. And so let us begin with:

\section{The atomic nature}

Considering all the phenomena in nature what parts will join with one another as well as stay aloof from one another? Our answer would be "Magnets." But why just magnets when molecules also consist of parts holding onto one another, or we ourselves to mother earth? We are not magnetic yet it is by magnetic power that we are held to the earth. (Ref-1) Gravity is but an inclination, the power of it belongs to magnetic, for we are not drawn to just the earth, but to its very center, and only our magnetic force provides the earth with that center. Two plus two therefore adds to four.

Then there is that electrical that we have placed in the atoms, giving it positive and negative parts as if these had an attraction to one another like unto the polarities of a magnet. But boy how wrong we are since that electrical all in itself in every possible way clearly shows us that its positive never at all attracts itself to any negative nor visa versa, On the contrary, these when confronted with one another will come to destroy one another.(Ref-2) That thus eliminates any electrical in the atom and more than anything whereby they may join into molecules.

Then we decided to invent a new bird called "electromagnetic" to somehow justify that positive to adhere to the negative in the atom. But once one has given himself over a cliff he is not likely to make it look like as if he is not falling, gravity will not let him. If anything we should have replaced those electrical polarities with the magnetic notations of north and south. But then gravity will accelerate us down from that cliff having to admit the atom to have a magnetic nature. And it's a sin to agree with me. But what is one sin more than another; the atom as a planetary system is so widely accepted why should we wish to change it? But it does demand an answer to two big questions, how any one planetary system can possibly join with another? And where are its parts as single sided coins coming from? 
Our solar system does not work electrical, it is made up of full fledged magnets; all planets as well as moons are full magnets along with the sun having that unique capacity to hold onto one another. Yet none of them cling at any close range, even while the sun pulls on all of them with its grant magnetic coordinate. These planets then keep themselves from falling into the sun by their velocity presenting sufficient inertia to balance out against the pull.

\section{The magnetic moon.}

It has been said or believed that our moon does not have a magnetic force like unto our earth. But that is self contradictory when we do give that moon a gravitational force of about $1 / 6^{\text {th }}$ of the earth since it is a confirmed fact that gravity cannot exist without it's magnetic man. Then there are the tides to refute it as these come about by the magnetic tentacles of the moon (Ref-3) And no less Newton's law of motion showing gravity to be a local force with a very limited reach. (Ref-4) Conclusively we have to keep our story straight.

\section{Why positive to negative show no attraction}

Has anyone ever scrutinized the electrical potential? How for example when a wire is held next to a magnet that it will push and pull itself to that magnet at the rate of its voltage, it's rate of rotation? And what does that prove if not that electricity is nothing other than a magnetic potential having a north and south polarity at its sides that as such rotate. And no wonder that it rotates because we are causing those rotations with the armature of our generators as we twist the straight magnetic lines of the field coils over one another to become twisted magnetic lines. (Ref-5)

And because the fundamental nature of magnetic is "curvature," each single one of these twists show themselves as any magnet shows itself, full figure eights of force. But at its ends that electricity is not that peaceful for while that whole circuit rotates in but a single direction of rotation - when we curve the two end to face each other (positive to negative) these show themselves in opposite direction of rotation that by contact destroy one another. We however found a way around it by adding turns in between to serve as a transmission between the two. That transmission then serves us for motors and transformers, as well as light and heating elements.

While thus it is a fact that there is a push pull from the electrical, it is only so at its sides, not at its connecting ends. (Ref-2) Nor therefore can these sides do what magnets are known for since these at all times rotate. They can however cause to vibrate.

Nor is electricity electrical but magnetic, twins may have different names but their parents are the same. And for a word in wisdom, there is movement and movement, but for all that is motion - there are but two directions of movement linear and angular, straight or around, and that by any combination. Since then these are most fundamental there is angular inertia, and linear inertia both of which weigh upon us for gravity. And no less for our movements unless one wishes to add up and down, both of which are still linear. Waves likewise in the way they travel are both angular and linear.

Nature is three-dimensional, with motion being singular having two directions of, then again most everything in nature is twofold starting with ourselves as man and woman. But this thing we must get away from, to think of electricity as electrical. Magnetic is like stationary, while electric is magnetic the same but in rotation. As then the atom is magnetic yet rotates shall we therefore call it electrical? I suppose we may have a point, but no that is not the right way to do things, I just got through saying that electrical is magnetic all the same, as in fact that rotating magnetic is a derivative of the regular magnetic. So no! We do not have a point - the atom as a rotating magnet is still magnetic, only when we twist the lines of magnetic are we allowed to keep the term of electric. 


\section{Electromagnetic}

If electric is to be thought of different why not for electromagnetic, for that is like saying "magneticmagnetic". And so what shall I say? I heard you the first time, why the repetition? Magnetic once is enough, for that is the reality of electromagnetic, a bird that does not exist, calling it twice by the same name does not make it a different bird, nor even two birds. The pronunciation of that so-called word or thing is the same as saying "turningstraight", or "angularlinear". The spell checker turns it down as not spelled right, it's supposed to be two words, the two directions of the single movement. The reality is magnetic either straight or angular, either stationary or rotating. No educated person will believe upon single sided coins, very well knowing how these things aside of not existing, cannot possibly exist. Wherefore protons and electrons never even made it to the atom.

\section{Nature of energy}

I should make a quotation: "In physics, energy is the quantitative property that must be transferred to an object in order to perform work on, or to heat, the object. Energy is a conserved quantity; the law of conservation of energy states that energy can be converted in form, but not created or destroyed."

We do have an idea - but no statement as to what energy really is, and how am I to make it real for us? If I say that the spark on the top of a cylinder is energy, it will make no sense. So thus let us go to a golf course. Here is the ball on the ground, it has no energy, it is just a ball on the ground. But then with a club we smite upon it to the ninth hole, and yes now it has energy because it is moving, it for all its energy can even kill someone if it strikes him on the head. Its motion is its energy, and our striking at it was the transfer of our energy upon it.

When you see gasoline as a liquid it has no energy, it's just a liquid good for nothing unless we add energy to it. We cannot really turn that gasoline into energy, yet we can produce energy by it. But what talk is this of me to keep saying energy, when it is always motion, and motion only, for that is what we are after, we want motion; the motion of the piston moving down, we could care less about the rest, as long as that piston moves we have what we want.

And so let us put some gasoline on top of that cylinder. Then to ignite it we use a spark, but nothing happens, why not? We are prone to say that its energy wont release for us, but of course not, it does not contain energy, its just a liquid or gas, and even though we did introduce some energy, namely the spark, it refused to work to our satisfaction. But how was that spark energy? It was so because it was a movement, a high movement in rotation, namely our so-called electricity that is nothing more than a flow of magnetic at high speed. In order thus to have energy something has to move because only movement is energy, "it" only has the ability to work, to perform work.

How therefore are we to make that liquid or gas alive to provide us with movement? The atoms of that liquid are formed into molecules and they rotate, and that rotation as such is energy because rotation is a movement. If then we can increase the speed of that rotation, their magnetic fields will come to expand, and that expansion being movement adds yet more movement demanding more room that in turn moves the piston down. Since then the spark alone did not do the trick other than to warm it up a little, we need an additive. And God created just such a thing called the oxygen atom/molecule, a very peculiar atom to form in pairs with a coordinate perfectly suited to make things alive that is to cause to restructure and or rearrange molecular formations, also said; to react with. With that additive the reaction by and with the high movement of the spark comes to increase the rotational speed of the atoms of the gaseous molecules at the same instant when the gas molecules are broken up forming $\mathrm{CO} 2$ and $\mathrm{H} 2 \mathrm{O}$. 


\section{Cornerstone}

The process starts with motion, - produces motion, - and ends with motion. In order to obtain movement it must be instigated by the same, like hitting that golf ball, human power transferred upon a ball. Or that spark as a high rate of conductive movement instigating a chain reaction at essentially the speed of light, first raising the rate of the oxygen atoms that then by its conductive movements conducts it upon the gas molecules whereby these are broken up, normally called combustion. It is not only in the break up of the molecules that these demand more room. (The smaller part of the expansion.) The primary demand for more room is by the atoms themselves as these are near instantly driven to a high rotation. And their magnetic fields rotating with them these expand driving each and every atom from one another.

The best part in gasoline thus are the hydrogen atoms since the lighter the elements so much the greater the expansion will be. And it's because of its conductive nature that one movement at high speed brings another movement to high speed, and that to us is like in an instant, near instant acceleration.

I placed this heading as: "Cornerstone." And that holds true for most everything whereby a great deal of energy (motion) is released. For a ten story building resting on a cornerstone the power of the destructive force is not in the cornerstone, but in the collapse of the building. Equally so with the atomic or hydrogen bomb the uranium, is like the cornerstone, or like the spark in the cylinder also being a cornerstone. The greatest of all fires can be started with but a single spark. Wherefore it is not in a small amount of uranium where all the power or energy resides, but in what is instigated by them to their surroundings. It is not the uranium to level a city, but the superheated air. Or a quake in the ocean that does no more than setup a water wave, that wave then when like a bear driven into a corner, will come out fighting - to level the seashore.

Energy is never anything but movement, as then it is and can be transferred it is never conserved. Man's law pertaining to that is quite wrong. Motion can be started and stopped without being transferred. A vehicle can move as well as be idle, its reduction in movement then is not transferred to the brakes, since these will cool down, and be like before. The same for warmth when cooler air comes to lower the rotational movement of the atoms in the warmer air by their conductive nature they are simply slowed down, nothing is conserved. The sunlight upon the earth is never conserved, it instigates warmth and growth, but that will slow down again and end their growth. Motion can be sped up and slowed with or without conservation, be it the wind or the atom or molecule in their rotation.

There is but one movement that never slows and can never be slowed down, nor even be reacted upon by anything, yet it reacts upon everything as it powers all nature, the one great fundamental movement that is an entity in itself, while no other motion nor movement being so fundamental, but derivatives of the first fundamental entity, that is ever directionally conductive the nature of which is best known as magnetic, with my notation for it as $3 \mathrm{M}$.

\section{Forces}

How many forces do we know? The wind, the water, man's strength, and so there are more to be named. But none of these rank among the fundamental ones, the ones to provide for the first of all motion. "The first of all motion"; so I said, for there is no other force than motion, there exist no other force than motion. Reality, so I aim to impress upon everyone. What is the sense of a strong force or a weak force, or even a nuclear force, when no name nor composition, nor nomenclature is given? It means nothing to say "strong" when the name of that gorilla is not given. And so I surmised we have no idea what in fact the fundamental forces in nature are, or else we would not have said, strong nor weak, nor nuclear.

As then I spoke of two things fundamental I named but one, and for good reason. But why should I name it when it should not be of me. And so; think of anything that is power or force, anything at all with the ability to 
procure things, to perform work, to get things moving, or to hold things together. What is our answer? Our own dictionary gives us the answer, namely "Motion."

There is nothing whatsoever in or upon the atom, or molecule to bind them together than the simplicity of motion. No other force or entity exists. But of course I must now reveal this wisdom that - that particular motion, is not just any motion, but something most fundamental to be an entity in itself. An always existing movement that cannot be removed, nor slowed down, nor sped up. It starts out with eternity, the meaning of which is something without beginning and without end that I may illustrate by a circle. The movement in a circle always returns to where it started. There is no entry in a circle, no opening, and it shows or contains but one single direction, the angular format, the linear that we know so well is missing. Therefore in order to have birth of it - when that circle is turned by one half it comes to resemble the figure of eight, the typical format of magnetic, that then gives birth to linear movement. (Ref-2)

But what comes next is equally in wisdom, that this most fundamental movement is conductive, and at that directionally conductive - wherefore, and whereby magnets are drawn together as well as draw away from each other. I then am speaking of magnetism; for that is the term we have given it. I then gave it the notation $3 \mathrm{M}$. I had people telling me that I was wrong in asserting that the format of magnetic is by an eight rather than two circles, even though I presenting undeniable evidence; the tides upon the earth is one such. (Ref-3) I know the old saying that one can lead a horse to water but not make him drink, if then we die of thirst do not blame me.

Contrary to some I at least am providing the full nomenclature of the force that I am placing upon the atom, name inclusive. A name that we all know for a force, a movement that presents linear as well as angular movement, with this obvious line between the lines that there is no other direction of movement. All things essentially being dual or twofold it stands to reason that it might be so for the fundamental of all things as well. And yes so it is, but first let us grasp what can be understood before we dive too deep where one might get the bends.

Does it not make good sense for that fundamental motion to exist just as I have said? It is found with all magnets and with all the waves of the spectrum, and no less for its derivative called electric. That always existing movement did not just fall out of the sky, it was created by Him who made the countless many stars. To Him belongs the honor and my honor.

\section{Expansion}

We know what expansion is, and sometimes see it, it appears as steam, a drop of water into many times its volume. But more obvious are those high power lines strung all over the country that for the high rotation of their magnetic field must be hung on long porcelain holders. But no less for a high rotation of the magnetic fields of the atoms of the water molecule as to how and why steam comes to take up so much volume. The one and only way whereby any spinning atom is able to demand more room is with and by a magnetic field, that typical figure eight of force.

Expansion is also seen in ice from water, and though it is from and by magnetic - that expansion is not in the rate of rotation, nor by the reduction in their rate of rotation, but simply in the formation of the grid. When baking bread or making pancakes the increased rate of the atomic movement turns liquid into a somewhat expanded solid. Add baking powder and buttermilk and it will become fluffy, adding air holes within the grid. Rates of movement restructures molecules and turns them into grids, but it can also destroy grids, for so there are ten thousand and one variations all by rates of movement. Since then nature shows itself in the nature of magnetic - why not accept it as such? 


\section{Rate of movement}

Have we ever wondered how or why water turns into ice, or steam, and even separate into its two components? And how and by what these two components come to reunite again? This one example in itself tells us a lot about nature in how and why it comes about. At room temperature those three atoms hold together by their magnetic movements, all atoms indeed being like unto magnets, but very special magnets, more unique than anything I can possibly name. Figure 1 is a crude illustration of one of the simplest of atoms with figure 3 a representation of our familiar $\mathrm{H} 2 \mathrm{O}$. A regular magnet placed on a table presents but a single movement, the $3 \mathrm{M}$, while with the atom there are always 2 to $\mathbf{4}$ forms of movement associated with it. The $3 \mathrm{M}$ in figure 1 is its one and only fundamental movement the typical figure eight of movement that as such is classified magnetic. Additionally that atom may or may not spin, but when it does, or let me say, is allowed to spin; or forced to spin; that format of $3 \mathrm{M}$ rotates along with it even when it is joined with others. That movement - or energy if you will - is not classified fundamental, nor any of its further movements, like a vibration, or oscillation when push/pulled upon, or turning along with the whole of the molecule.

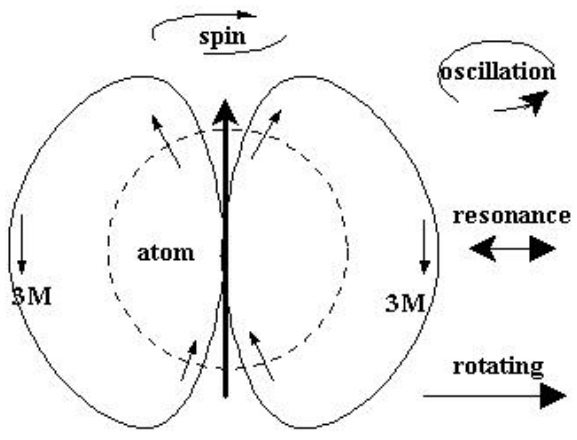

Figure 1: From one to five movements associated with the atom.

Atoms at different temperatures have the ability to mask their polarities, how precisely is a good question, I know only a little of it. But this much is sure when the rate of movement upon the water molecule is increased; the $3 \mathrm{M}$ of each of them expanding - it consequently drives the 3 atoms further from one another, the liquid taking on a gaseous form known as steam. And with still higher and higher velocities their fields are stretched so far to break loose from one another. Normally we speak of heating, since then heat is movement in the rate thereof, motion can be used as another term for heat.

It took a great deal of heat to break up the water molecule into its two components, but it takes an even higher rate of motion to recombine them. The spark or flame that will instigate the reunion has a much greater velocity that at virtually the speed of light instigates a chain reaction from atom to atom raising their rotational speed to its own speed, with this result greatly increasing their $3 \mathrm{M}$ (magnetic field). The hydrogen bomb is evidence to that effect.

Those two hydrogen atoms then can also sliced off from that oxygen atom without any heat by a rotating magnetic field, that is to say, between the two ends of a rotating magnetic field also known as electricity. Here the two ends of that circuit turning in opposite rotation of one another have a neat way to slice atom from atom, to separate water into its two components. If now only we could do that at the top of a cylinder without blowing ourselves up, we could use water for a fuel, more potent than gasoline.

I call it curious that at such extreme expansion the two components reunite two to one, and immediately afterwards their rate of movement drops down to what we perceive as room temperature; mere $\mathrm{H} 2 \mathrm{O}$. (Conservation is none existent here) When they were together the high rate broke up their love scene, while the still higher rate did reunite them. As then we may not quite understand that, it's nonetheless a fact in 
nature seen in various ways. One is with the hydrogen bomb, the element of hydrogen being the perfect agent by which to obtain a great deal of energy, it being the lightest has the greatest expansion of all. The more hydrogen we can cram into a fuel so much more downward push we can have at the pistons. Joining hydrogen to oxygen is more powerful than any gasoline. I learned that the hard way in my experiments.

But now comes an even more curious part how when the rate of movement upon these 3 atoms is slowed down to what we call freezing - these little rascals alter their magnetic polarities whereby to lock onto other molecules forming into grids known as ice. Just previously the directions of their magnetic polarity held them together very well while masking their adhesive power to all other atoms. And thus by no more than slowing down that internal movement, (their spin along with the $3 \mathrm{M}_{1}$ ) shifted the overall magnetic coordinate enabling the oxygen to instead of two points - present three points whereby to adhere to others, and each hydrogen atom opened up like unto regular magnets.

All atoms then at all times have the ability to adhere at four points, its north and south along with the two equatorial regions. And that can be increased to six points vertical and horizontal, and that while it spins obvious in all types of grids. That oxygen atom in ice has 4 points in the horizontal, 3 of them adhering to other atoms while number 4 keeps it aloof from others creating those openings in the grid, whereby ice takes up a little more room, illustrated by figure 2 . Additionally these presents 2 more points in the vertical. Note the polarity in all of them to form such grids, the six oxygen atoms driving themselves into a hexagon by like polarities.

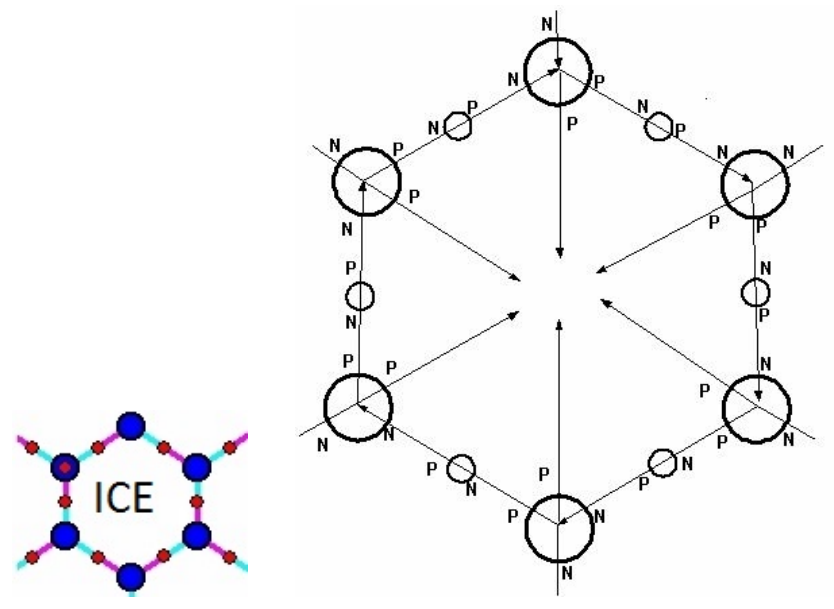

Figure 2: $\mathrm{H} 2 \mathrm{O}$ by magnetic power formed to a grid

So what do we think of our oxygen atom to present itself with six points around the sphere of it - able to join and/or maintain itself at a distance from others, and yet again like in air mask all of its polarities to adhere to none other. It does all that by various rates of movement, that yes, and we must include its surroundings, by what type of atoms it is next to, or in contact with. When it meets silicon atoms the oxygen atom comes to appear like regular magnets, and while our oxygen atom in the air shows no polarities, yet it takes but a tiny spark (a high movement) for it to open up and restructure other molecules like those of benzene etc.

A most remarkable atom that one of oxygen, more than any atom that I would love to have a full understanding of. If anyone is able to show just how these molecules either mask and shift their magnetic polarities to perform all these feats, I am all ears, for to me the atom is most astounding to as magnetic agents form all that is in nature. And it cannot be anything else in nature, seeing no force nor movement is as unique as the eight of magnetic. No strong, nor weak, nor any other force is needed to perform what the eight of magnetic is capable of. It quells everything within its embrace, maintaining a strong solid atom without any other part or force needed. 
Since then these appear in a whole variety of formats from single to multiples in one, so it is that butter is parted at the touch while steel must be cut by others of like complexity. The atom of steel is another fascinating subject, how it for its complex magnetic formation hold so well together that when heated - its fields attempting to expand are prevented by its own complex nature, or coordinate as I should say. Elements like gold and silver, or copper etc, do not serve well for a fuel only because these do not expand as the lighter elements. But then these serve another purpose also needed. Accordingly it is each for its own to serve all. A wisdom we often forget.

\section{The H2O Molecule}

Figure 3 illustrates the molecule of water as I visualize its coordinate. Is then the atom of oxygen in water single or dual? For how in air being dual does it come to be single at other times? And by 3 in one it is poisonous by virtue of its now new coordinate that is harmful to humans. One hydrogen atom now connects with an outgoing movement while the second by its ingoing movement. Since then it seems to me that there should be some polarity open for others to connect to at the bottom ends of the two hydrogen atoms reality shows me in error. Only when their overall movement is slowed down to the point of freezing do these ends join, otherwise they are masked preventing other molecules from joining with it. The ideal at the top of the oxygen atom/molecule being masked is more easily understood, in that by their dual nature in air when it is in the company of nitrogen atom/molecules are always masked. Yet when confronted with silicone and many other elements it readily reacts, but not without some instigation. But then again as it is transported by our bloodstream it reacts with the cells in our bodies without any apparent instigation. If then we truly wish to understand nature stick with magnetic in the atom also since electrical or anything else would destroy the atom, or in the first place never come to exist. And yes I do know more but let us first grasp what is real in nature.

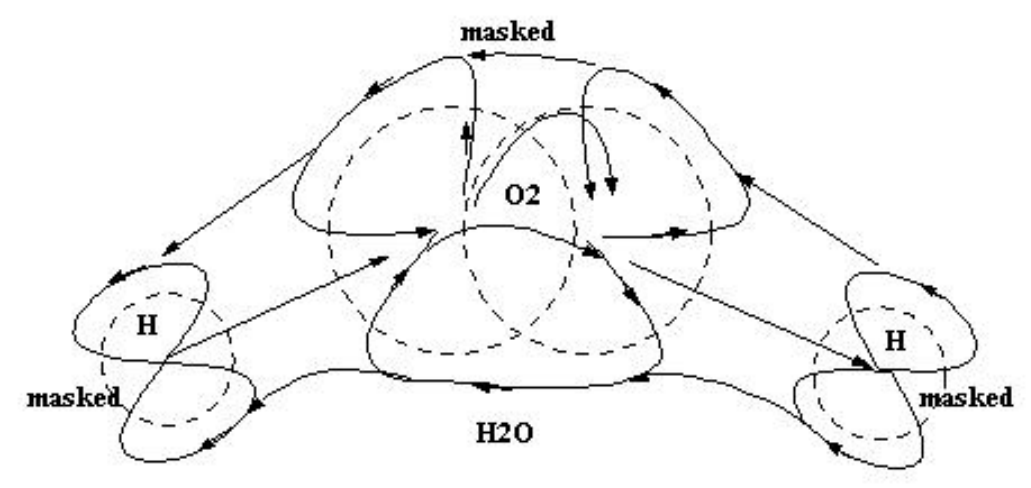

Figure 3. The water molecule after its lines of conductive movements.

And so how shall we redraw this molecule in the nature of magnetic? Who is competent enough to understand the atom as it really is in its many forms and coordinates to perform all that is found in nature? No doubt an endless subject but most rewarding, I for one am at its threshold, as yet from the outside looking in.

\section{What is a charge?}

Shall we call it mis-apprehension or that the Almighty One withheld knowledge from us because we did not honor Him as He is to be honored for our very life and being? We have the habit of saying; that something is charged. That is like saying how Henry took it from me, but - "what" - did Henry take from me, was it green cheese or a Martian? For if we cannot specify what a charge is, we ought not pronounce the word. If acceleration creates a charge then the acceleration "is" the charge, since to accelerate is an increase in a rate of movement. That thus comes down to motion, the motion of the part, and how does that create what we 
fail to mention? If somehow we come to mention electricity, it takes two to tangle, where thus are the lines of power to be twisted over one another? It takes rotation to come to twist, and twist what?

And how is it that we come to say that "acceleration" creates a charge? Will it ever come to a stop? Must not that which accelerates come to a final? According to our vocabulary these parts forever accelerating must now be traveling at a trillion times the speed of light.

And why should we even want any electricity in the atom when as a standing entity it does not do anything, like as if it does not even exist. But once the circle is completed it will destroy itself unless we appease them with a transmission, that for its nature can only be done by turns and more turns, either an already rotating movement or physical turns. (Ref-5) Where then is that transmission within the atom? We are better off with a magnetic nature, even though it may be difficult to grasp, it's the only thing available whereby nature may be formed. Any other theory or ideal is impossible to grasp or even come upon.

\section{Single sided coins}

If a particle moves or accelerates it has a coming and going does it not? Or anything that moves has a coming and going that as such we came to look upon as positive for the one end, with negative for its other end. It does not matter which end is called which, since these terms are no more than a point of view for a single entity. Not just any entity in that for magnets it is called north and south, while our chair has a top and bottom. Our legs then are beneath with our head held upwards. But how is one to have any single thing with just one end, or a sheet of paper with no bottom? Think about it and forget about only positive or only negative, seeing there has never been such a thing, nor ever will be. Each term exists because it is simultaneous and in conjunction with the other of a single entity.

\section{Power of magnetic}

Figure 4 illustrates a molecule of gasoline consisting of 5 carbon atoms with hydrogen atoms bonded to the outer 4, The quest then becomes how these bond to one another after the manner in nature where all things are by movement into movement by coordinates.
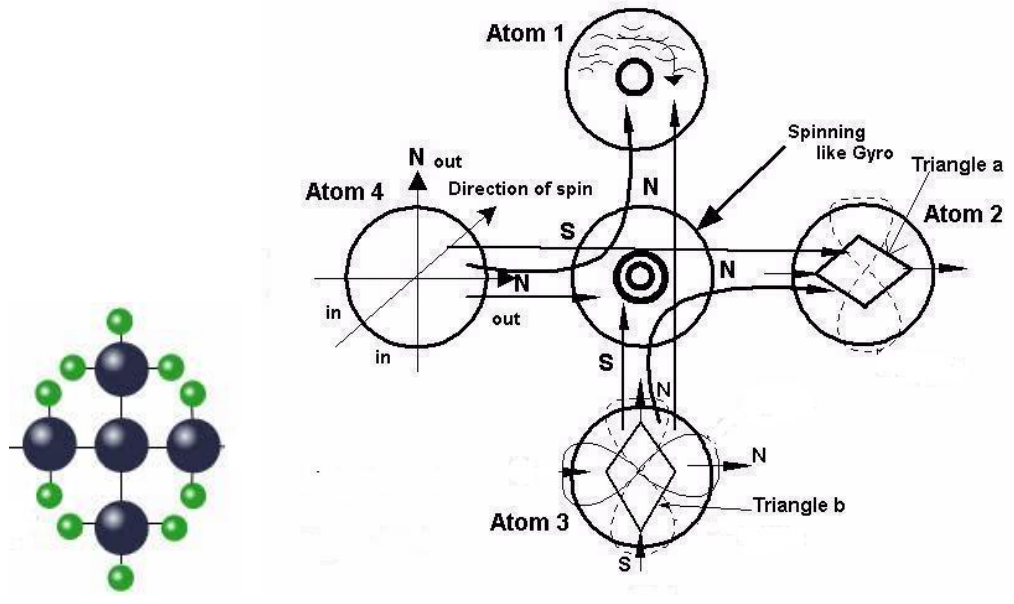

Figure 4 Gas molecule locked magnetically

And when an oxygen atom at high movement is directed at them it will break up that magnetic hold restructuring it. But the power By atom 3 the direction of its internal movement is incoming from the south and outgoing on the right and the top called the north. From here atom 3 with its conductive motion bonds 
directly to the center atom as well as to the right atom 2, as well as to atom 1, and again to atom 4. Each single atom thus helps to bond all five one to the other. In the overall view two of the five atoms act as a south, or negative passing over the center atom towards to the remaining two as were they the north, or positive end of this entire molecule.

of it all rests with the near instant expansion of their magnetic fields - during the restructuring - by the increase in their rotation. The "real" power in our internal combustion engines thus is "magnetic." Then consider the high current at those wires strung on those large power poles. That current not only rotates at the clip of a quarter million rotations (volts) each second, but reverses its rotation for the half thereof - also at the same rate. (AC current) How agile therefore magnetic is. And yet that rotation is but a snails pace compared to its linear velocity at $300,000 / \mathrm{km} / \mathrm{s}$. Consequently all the movement upon the atom is slow compared to the speed of its "Fundamental Movement," its - "first of all movement."

Then to have a look at the atom in figure 5, how complex can a magnetic field be? When we view or experiment with any of our available magnets, we are only dealing with the most simplest of all, usually showing no more than its first movement unless we put it in a drill to start rotating it. For this is what the atom does without a drill. But then I may be getting myself in hot water to get too deeply into how and why the atoms spins, or their fields upon them. I may have hinted at the twofold nature of just about everything in nature inclusive that most fundamental one called magnetic that in reality is a movement all its own, but then in going further I made an oath.

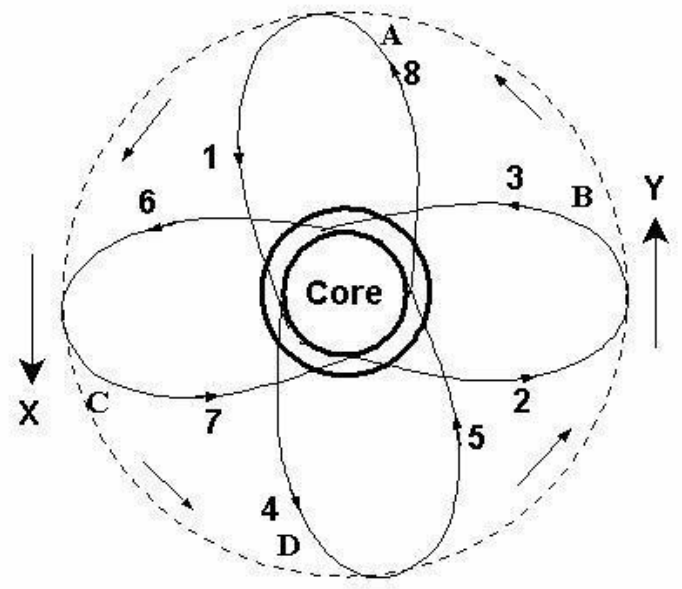

Figure 5. Atoms in multiple formation real, but hard to comprehend.

I did however wish to show the multiple coordinates that can and does exist upon many atoms fueled by the first of all movements. The illustration figure 5 appears like 2 figure eights offset by 90 degrees where from $A$ to $B$ the North with from $C$ to $D$ the south. Or if we follow the internal line of motion from 1 to 8 it is a single movement, one that always returns from where it started like any and all magnetic movements always do. One may be skeptical of the atom in figure 5 , but more complex variations can be drawn quite realistically.

When we look at the grids of many compound elements it's obvious how atoms can and will join at as many as 4 to 6 points simultaneously. That therefore cannot be done with any hydrogen atom, its coordinate being too simple. The internal structure of the larger atoms do present its coordinate in what appears to us as multiples. And if by its design that coordinate becomes unstable it will work tirelessly to rectify itself. It then does so mostly with the help of other coordinates it can come in contact with. If then our oxygen and nitrogen 
atoms in their dual nature were unable to mask their polarities to others our air would be solid - as it is when cooled to a very low temperature.

And what more is there to say in this endless subject to get to know the atom? No doubt many will work on it to man's gain, I have only made a start.

\section{Conclusion}

The atom for its magnetic nature may appear as a whole new concept, but it's the only concept of any reality. The many facts and observations in nature only disprove the atom as currently taught. If then we backtrack to simplicity we will find the magnetic atom a most rewarding subject.

\section{References}

Ref-1: https://cirworld.com/index.php/jap/article/view/8164

Ref-2: http://recentscientific.com/magnetic-and-electric

Ref-3: https://cirworld.com/index.php/jap/article/view/8114

Ref-4: http://gsjournal.net/Science-Journals/Essays/View/5863

Ref-5:http://gsjournal.net/Science-Journals/\%7B\$cat_name\%7D/View/6071 